\title{
Where there is no doctor: can volunteer community health workers in rural Uganda provide integrated community case management?
}

Jennifer L. Brenner ${ }^{1}$, Celestine Barigye ${ }^{2}$, Samuel Maling ${ }^{3}$, Jerome Kabakyenga ${ }^{3}$, Alberto Nettel-Aguirre ${ }^{1}$, Denise Buchner ${ }^{1}$, Teddy Kyomuhangi ${ }^{3}$, Carolyn Pim, Kathryn Wotton ${ }^{4}$, Natukwatsa Amon ${ }^{3}$, Nalini Singhal ${ }^{1}$

1. University of Calgary, Department of Paediatrics, Cumming School of Medicine

2. Ministry of Health Uganda,

3. Mbarara University of Science and Technology, Maternal, Newborn and Child Health Institute

4. Mbarara University of Science and Technology Faculty of Medicine, Community Health

\begin{abstract}
Introduction: Integrated community case management (iCCM) involves assessment and treatment of common childhood illnesses by community health workers (CHWs). Evaluation of a new Ugandan iCCM program is needed.

Objectives: The objectives of this study were to assess if $\mathrm{iCCM}$ by lay volunteer CHWs is feasible and if iCCM would increase proportions of children treated for fever, pneumonia, and diarrhoea in rural Uganda.

Methods: This pre/post study used a quasi-experimental design and non-intervention comparison community. CHWs were selected, trained, and equipped to assess and treat children under five years with signs of the three illnesses. Evaluation included CHW-patient encounter record review plus analysis of pre/post household surveys.

Results: 196 iCCM-trained CHWs reported 6,276 sick child assessments (45\% fever, 46\% pneumonia, 9\% diarrhoea). 93\% of cases were managed according to algorithm recommendations. Absolute proportions of children receiving treatment significantly increased post-intervention: antimalarial for fever ( $+24 \%$ intervention versus $+4 \%$ control) and oral rehydration salts $/$ zinc for diarrhoea $(+14 \%$ intervention versus $+1 \%$ control).

Conclusion: In our limited-resource, rural Ugandan setting, iCCM involving lay CHWs was feasible and significantly increased the proportion of young children treated for malaria and diarrhoea.
\end{abstract}

Keywords: Uganda; maternal health; child health; community health worker; integrated community case management.

DOI: https://dx.doi.org/10.4314/ahs.v17i1.29

Cite as: Brenner JL, Barigye C, Maling S, Kabakyenga J, Nettel-Aguirre A, Buchner D, Kyomubangi T, Pim C, Wotton K, Amon N, Singhal $N$. Where there is no doctor: can volunteer community health workers in rural Uganda provide integrated community case management? Afri Health Sci. 2017;17(1): 237-246. bttps://dx.doi.org/10.4314/abs.v17i1.29

\section{Introduction}

Every year, 6.6 million children under age five (U5) die worldwide, mainly from diseases that can be easily treated or prevented. ${ }^{1}$ In rural sub-Saharan Africa, child mortality is especially high and aggravated by severe shortage of health professionals. ${ }^{2}$ Early and correct treatment for top killer diseases is essential to reduce child mortality where facility-based services alone cannot provide adequate services. ${ }^{3}$ Community case management (CCM) involves treatment of selected common childhood diseases by lay community health workers (CHWs). Integrated community case management (iCCM) involves assessment and

\section{Corresponding author: \\ Brenner Jennifer, University of Calgary, Department of Paediatrics, Cumming School of Medicine \\ Email: jennbrenner@me.com}

African Health Sciences Vol 17 Issue 1, March, 2017 treatment of more than one illness, using algorithms to manage uncomplicated illnesses including malaria, pneumonia, and diarrhoea. Currently, governments throughout sub-Saharan Africa are scaling up a variety of CCM and iCCM programs. ${ }^{4-9}$

During the past three decades, national CCM programs in countries such as Nepal, Pakistan, Honduras, and Senegal have targeted specific (and often single) diseases, with partial CCM coverage in over forty other countries. ${ }^{10}$ Key CCM program and literature reviews highlight the wide-variety of program models used globally and suggest that with adequate training, a consistent drug supply, and supervisory support, lay CHWs can adequately manage single illness treatment and impact health outcomes. ${ }^{2,10-14}$ Three illnesses with strong CCM evidence are diarrhoea, pneumonia and malaria. ${ }^{11}$

ICCM builds on CCM success through training and equipping CHWs to identify and manage more than one con- 
dition, depending on the child's presentation. ${ }^{3}$ ICCM recognizes that a single child may be afflicted with multiple illnesses at one time or may present with symptoms that could represent more than one possible diagnosis. ${ }^{13}$ However, a potential concern is that the ability and willingness of lay (especially volunteer) health workers to undertake multiple responsibilities could potentially compromise care. ${ }^{15}$ In Asia, Pakistan and Nepal have well-established and studied iCCM programs. ${ }^{16,17}$ African iCCM studies are few, ${ }^{14,18}$ and less still have assessed health outputs and outcomes. ${ }^{5,14,19-23}$ National programs in sub-Saharan Africa are new and while early studies are demonstrating implementation feasibility and early positive outcomes ${ }^{7,8}$, more studies are needed to understand implementation in a variety of contexts including use of volunteer CHWs with limited formal education and short iCCM training.

Uganda has high child mortality ${ }^{1}$ hampered by difficult access to care, especially by rural and poor families. ${ }^{24}$ Several years ago, Uganda established a national CHW program involving 'Village Health Teams', which formalized training and job expectations for volunteer CHWs as a recognized health system cadre. ${ }^{25,26}$ Building on this program, a country-wide iCCM strategy was launched in 2010. ${ }^{6}$ An evidence-based algorithm directed CHW treatment with anti-malarial drugs for fever, antibiotics for presumed pneumonia, and oral rehydration salts (ORS) and zinc for diarrhoea. Guidelines outlined iCCM CHW selection, training, and equipment. ${ }^{6}$ The Ugandan Ministry of Health hopes to soon implement iCCM nationally, however, the program has yet to reach scale. 'Best practise' evidence on training, monitoring, and evaluation is urgently needed. Although several iCCM study projects are underway, only one group from Eastern Uganda has published intervention outcomes and operational data thus far, specifically demonstrating feasible CHW management using a dual illness algorithm, ${ }^{27}$ an increase in care-seeking by rural families with access to iCCM, ${ }^{28}$ a sustained quality of care by CHWs using iCCM compared to single-illness management, ${ }^{29}$ a sustained adherence to dual illness medicines by families under iCCM compared to single illness treatments, ${ }^{30}$ and an increase in prompt and appropriate treatment of pneumonia under iCCM. ${ }^{23}$

Healthy Child Uganda (HCU) is a Ugandan-Canadian university partnership that has developed, implemented, and evaluated community-based maternal and child health initiatives involving volunteer CHWs for over a decade. We describe an implementation study using a quasi-experimental design (pre/post intervention with comparison group) to understand the potential of Uganda's newly proposed national iCCM program in rural SouthWestern communities. Study objectives included assessment of feasibility of iCCM provision by lay trained volunteer CHWs (process outcomes) and assessment of pre/post change in proportion of sick U5 children treated for fever, presumed pneumonia, and diarrhoea (effectiveness outcome) in this study.

\section{Methods}

\section{Study area and population}

The study was conducted in Bushenyi District in SouthWestern Uganda where Mbarara University of Science and Technology (MUST) has established relationships. The district is rural and hilly with numerous crater lakes. The estimated district population of $241,500(20.2 \%$ under five (U5) children ${ }^{31}$ lives in 'parishes' each comprised of about 10 villages (population $~ 300-600$ people) with larger 'sub-counties' encompassing collections of adjacent parishes. Most families depend on subsistence farming and many live in extreme poverty. Roads are few and mostly unpaved, and access to health facilities beyond some primary health care facilities is difficult. Government health facilities face staffing, infrastructure, and equipment shortages.

\section{Study design}

This implementation study was a prospective intervention trial with a non-randomized comparison control group (Figure 1). Sub-counties were chosen as control and intervention units to accommodate budgets, logistics, and supervision for this study. Two of 7 Bushenyi sub-counties were purposefully selected with district and investigator input. Kyabugimbi sub-county (population $\sim 34,500$ ) was chosen as the intervention area due to high (U5) morbidity and mortality according to district records, and difficult health service access. Adjacent Kakanju sub-county (population $\sim 23,300$ ) was chosen for comparison due to similar population size, illness burden, geography, tribal composition and remote location. Due to a river dividing the sub-counties, usual health and social service access is separate, potentially reducing cross-contamination.

During the study, control communities received standard care from existing government and non-governmental providers only, including primary and secondary illness management by nurses, midwives, and clinical officers, but rarely medical doctors.

African Health Sciences Vol 17 Issue 1, March, 2017 


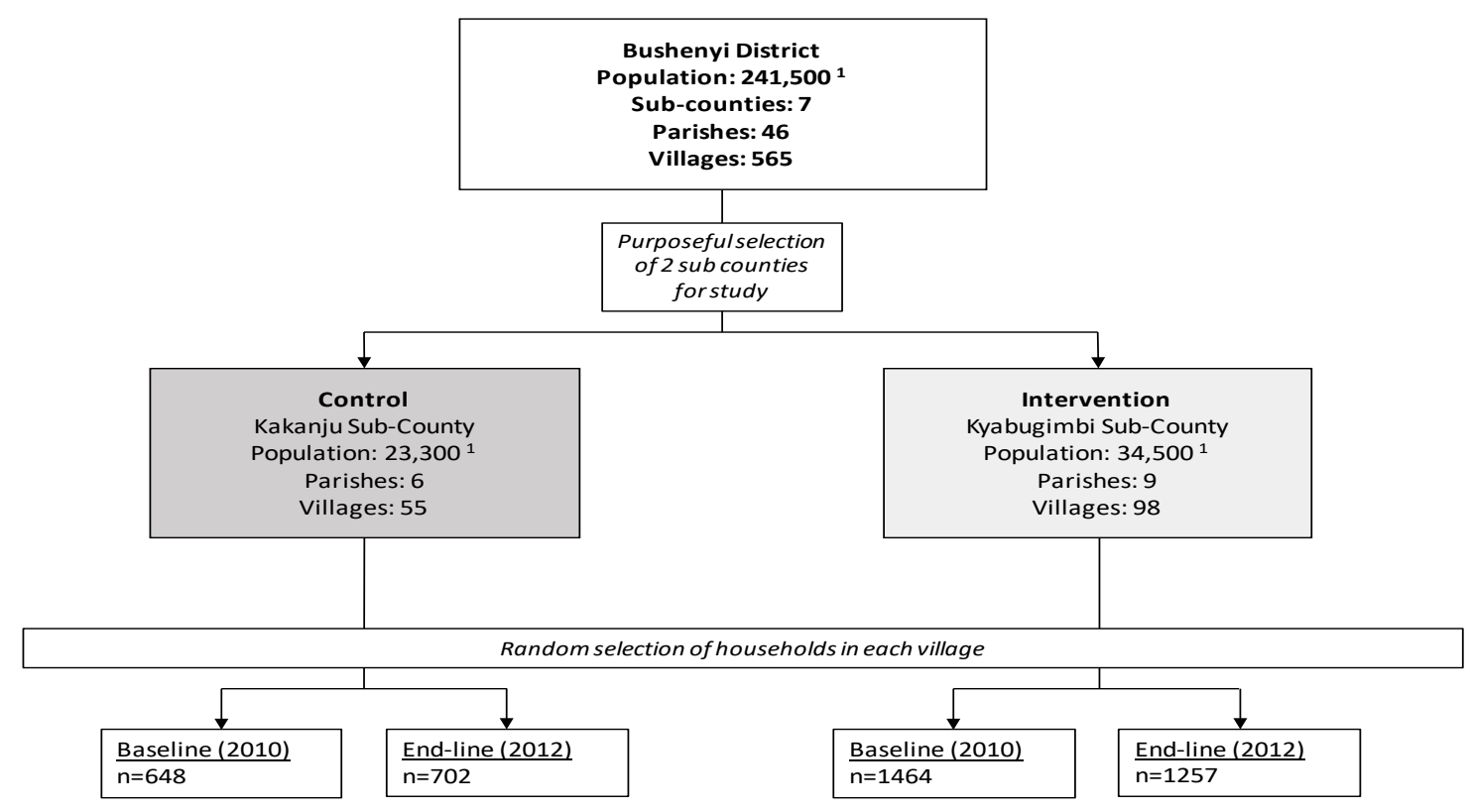

Figure 1. Household survey study diagram illustrating control and intervention allocation.

Note: $n=$ number of mother/household surveys analyzed.

${ }_{1}^{1}$ Population figures are estimates for 2010 obtained from the Bushenyi District Health Records database, calculated based on 2002 Ugandan national census data.

Care-seeking from traditional providers was common. Prior to the study, control or intervention areas did not have any selected or trained CHWs.

In intervention communities, CHWs were trained and equipped to provide basic health promotion and $\mathrm{iCCM}$ (i.e. 'iCCM CHWs').

\section{Intervention}

Intervention was with iCCM guidelines and training materials aligned with new Ministry of Health standards, ${ }^{6}$ with activities coordinated and supported by district health providers. As an 'implementation' study, the intervention was integrated within the existing government health system. CHW training, supervision and provision of study materials (job aids, drug supplies, algorithms) were provided by the district/Ministry of Health in accordance with Ugandan government policy.

Initial CHW selection occurred (July-October 2010), during village-wide meetings through community-determined selection processes. One CHW was chosen per 25-30 households (i.e. 2-5 CHWs/village) and attended a five-day 'Basic CHW' course. Training was conducted by trained government health workers from local health facilities and involved review of basic CHW health promotion responsibilities such as reporting, pregnancy/ post-natal visits, and surveillance. Each $\mathrm{CHW}$ received a pen, manual, T-shirt, and register. The only remuneration was a daily transportation allowance per training day ( $\$ 1 \mathrm{USD})$. CHWs were organized into parish teams for trainings; CHW parish teams met monthly with their assigned supervisor from the local health facility. CHWs provided health promotion to their respective communities according to Ministry of Health recommendations. All basic CHWs underwent a three-day refresher course one year after initial training.

ICCM implementation began once draft training materials and drug kits became available. A significant and unexpected 16 month delay between time of baseline/ initial CHW training resulted from consideration and deliberation of national policy changes to the iCCM drug algorithm. Once supplies became available (early 2012), two CHWs from amongst all trained CHWs in each intervention village were chosen to receive additional 'iCCM' training. 'iCCM CHW' selection was conducted within CHW teams, often based on village geography and/or willingness for the responsibility. 'ICCM CHWs' underwent a five-day iCCM training which included sick child assessment; symptom/danger sign identification; support, treatment and/or referral; and record-keeping. Each 'iCCM CHW' received a canvas bag, job-aid, timer, and wooden medicine box with a starter supply of pre-packaged and colour-coded medicines in sealed single-treatment packages. iCCM algorithms ${ }^{6}$ instructed Coartem ${ }^{\circledR}$ (artemether/lumefantrine) for fever (4-59 month-olds); amoxicillin for cough plus fast breathing(2-59 month- 
olds); and ORS/zinc for diarrhoea (2-59 month-olds). All symptomatic infants under two months-old or children with danger signs were referred to health facilities.

CHWs provided free treatment; an expectation communicated consistently at trainings and community meetings. One-half of 'iCCM CHWs' received mobile phones and associated training (i.e. 'iCCM plus mobile CHWs') as part of a synergy pilot intervention described elsewhere, ${ }^{32,33}$.

From April-October 2012, CHWs conducted iCCM. Drug stocks were replenished at monthly CHW meetings by the local CHW supervisor or at local health centres if supplies were required between meeting times.

\section{Data sources}

Three quantitative data sources were assessed to better understand feasibility (i and ii below) and potential intervention effectiveness (iii, below).

(i) $\mathrm{CHW}$ Registry

A Microsoft ${ }^{\circledR}$ Excel $^{\mathrm{TM}}$ database tracked $\mathrm{CHW}$ sociodemographic data, sex, age, education and $\mathrm{iCCM} /$ non-iCCM status and tracked CHW attrition. 'Drop outs' were defined as those CHWs reported by their supervisors to be no longer reporting regularly and attending meetings between their initial CHW training data and October 2012 (intervention end).

\section{(ii) Patient Encounter Reports}

All CHWs recorded demographic and health indicators for their catchment. As part of the feasibility evaluation and to approximate volume and type of patient encounters, 'ICCM CHWs' additionally recorded each sick child encounter (age, sex, illness signs, breath rate, danger signs, treatment, referrals). Reports were submitted to supervisors monthly. 'iCCM plus mobile' $\mathrm{CHWs}$ submitted encounter reports through their mobile devices.

\section{(iii) Household Survey}

Mothers of young children were surveyed at baseline (2010) and endline (2012) in intervention and control communities. A multiple-choice questionnaire assessing demographics and recent child illness, care-seeking, and treatment was adapted, translated (into vernacular), back-translated, and field-tested based on a Malaria Consortium tool and used with permission. At baseline, eligible households (at least one child under 59 months and mother present) in each village were approached for survey using a random numbers table and village list. Trained research assistants verbally administered surveys.
The same randomization to select households and survey process was repeated at endline.

\section{Data entry and analysis}

(i) $C H W$ Registry

Updated quarterly, a retrospective registry analysis involved calculating $\mathrm{CHW}$ characteristic frequencies and categorizing and calculating frequencies for those CHWs who were no longer reported as active i.e. "dropouts".

(ii) Patient Encounter Reports

CHW register data was manually entered into a database and merged into SPSSTM with mobile phone data downloaded from a main server ${ }^{32}$ A retrospective analysis calculated frequencies and proportions of patient ages, illness classifications, and treatments. In analyzing illness classifications, children were classified according to all presenting symptoms; a single child could be classified with up to three illnesses. Treatment was 'appropriate' if consistent with the iCCM algorithm based on age and reported symptoms. Dosage/ treatment duration weren't included in our definition of 'appropriate'.

(iii) Household Survey

Data were manually entered and exported into SPSSTM then analyzed using ' $\mathrm{R}$ project for statistical computing' software. Main indicator proportions used Z-tests of difference in proportions. Corresponding confidence intervals used Wald's formula.

\section{Ethics statement}

Study approval was through Mbarara University of Science and Technology (MUST) Institutional Ethical Review Committee and University of Calgary Conjoint Health Research Ethics Board. All respondents provided informed consent prior to participation.

\section{Results \\ CHW demographics}

During the study period, $288 \mathrm{CHWs}$ were selected from 98 intervention villages and underwent 'Basic CHW' training (average 2.9 CHWs per village). Their average age was 36 years (range 20-63) and 70\% were female. CHW formal education varied; $3 \%$ had three years or less of primary (P3), 47\% had completed primary (P7), 45\% had some secondary education, and $2 \%$ had post-secondary education. In total, $196 \mathrm{CHWs}(72 \%$ female) participated in $\mathrm{iCCM}$ training and delivery and the remaining 92 individuals conducted only health promotion as 'Basic CHWs'. Age and education of 'iCCM CHWs' did not differ from those of 'Basic CHWs'.

African Health Sciences Vol 17 Issue 1, March, 2017 
Between initial training and October 2012 (end of intervention), 12 of the initial $288(4.2 \%)$ CHWs left their positions. All 12 'drop outs' were from the non-iCCM ('Basic CHW') group.

\section{Patient encounters}

Based on their reports, during the seven-month iCCM intervention, CHWs had a total of 6,276 sick child encounters (48\% female) for which 8,349 illnesses were classified (average 1.4 illnesses per encounter). On average, each CHW reported 4.6 children per month; $2 \%$ were $0-2$ months old, $19 \%$ were $2-12$ months old, and $78 \%$ were
1-5 years old. Table 1 shows the distribution of classified illnesses. Presumed pneumonia was most common (46.6\% of cases), followed by fever ( $44.7 \%$ of cases), and 'diarrhoea' ( $8.7 \%$ of cases). In the large majority of cases $(93 \%)$, CHWs reported treatment consistent with their reported clinical findings and the recommended algorithm for their categorization of illness. Self-reported consistency with the algorithm was highest for fever (95.5\%) and lowest for presumed pneumonia (90.1\%). On average, CHWs saw 2.7 cases of fever, 2.8 cases of presumed pneumonia, and 0.5 cases of diarrhoea each month.

Table 1. CHW assessment and treatment by case, showing illness classification and percentage of 'appropriate' treatment given according to CHW patient encounter reports.

\begin{tabular}{|c|c|c|c|}
\hline Classification & $\begin{array}{l}\text { Number } \\
\text { of cases }\end{array}$ & $\begin{array}{l}\text { Percentage } \\
\text { 'appropriate' treatment }\end{array}$ & $\begin{array}{l}\text { Mean number of cases } \\
\text { per VHT per month }\end{array}$ \\
\hline Fever $^{1}$ & 3,725 & $95.5 \%$ & 2.7 \\
\hline Presumed pneumonia $^{2,4}$ & 3,892 & $90.1 \%$ & 2.8 \\
\hline Diarrhoea $^{2}$ & 723 & $91.6 \%$ & 0.5 \\
\hline All cases ${ }^{3}$ & 8,340 & $92.7 \%$ & 6.0 \\
\hline
\end{tabular}

Note: A 'case' occurs each time a CHW classifies an illness in a child, according to the iCCM algorithms; a single child assessed may be assigned to more than one category of illness, therefore number of cases of illness may exceed total number of assessments by CHWs.

\footnotetext{
**'Appropriate' treatment is defined as CHW reported medication/treatment in agreement with iCCM algorithm for classified illness and age range. Fever $=$ Coartem; presumed pneumonia $=$ amoxicillin; diarrhoea $=$ ORS and zinc

${ }^{1}$ Cases in 4-59 month-old children

${ }^{2}$ Cases in 2-59 month-old children

${ }^{3}$ Cases in CHW treatment age range, by illness respectively

${ }^{4}$ Presumed pneumonia $=$ cough and fast breathing
}

\section{Recent sick child treatment by maternal report}

A total of 2,112 surveys (723 intervention, 648 control) at baseline and 1,959 surveys (1257 intervention, 702 control) at endline were analyzed. There were no major differences in maternal age, mean education level, sex, or age of children pre- and post-intervention or be- tween control and intervention groups (Table 2). Table 3 shows the proportion of ill children who reportedly received 'appropriate' treatment for their illness(es) based on symptoms reported by mothers. The percentage who received 'appropriate' fever and diarrhoea treatment increased significantly post-intervention in iCCM intervention areas but not in control communities. 
Table 2. Household and maternal characteristics at baseline and end line in control and intervention communities.

\begin{tabular}{|c|c|c|c|c|c|}
\hline \multirow[b]{2}{*}{ Category } & \multirow[b]{2}{*}{ Subcategory } & \multicolumn{2}{|c|}{ Baseline (2010) } & \multicolumn{2}{|c|}{ End line (2012) } \\
\hline & & Control & ICCM & Control & ICCM \\
\hline Number of households & & 648 & 1,464 & 702 & 1,257 \\
\hline Mean U5s per household & & $1.5 \pm 0.6$ & $1.4 \pm 0.6$ & $1.6 \pm 0.6$ & $1.6 \pm 0.6$ \\
\hline Mean maternal age & & $27.9 \pm 6.8$ & $29.0 \pm 7.2$ & $28.6 \pm 7.2$ & $29.1 \pm 6.8$ \\
\hline \multirow[t]{6}{*}{ Education $^{1}$} & $\begin{array}{l}\text { No formal } \\
\text { education }\end{array}$ & $\begin{array}{l}8.8 \% \\
(5.2,12.4)\end{array}$ & $\begin{array}{l}10.3 \% \\
(7.7,12.9)\end{array}$ & $\begin{array}{l}8.1 \% \\
(4.6,11.8)\end{array}$ & $\begin{array}{l}9.1 \% \\
(6.4,11.9)\end{array}$ \\
\hline & P1-P3 & $\begin{array}{l}7.9 \% \\
(4.3,11.5)\end{array}$ & $\begin{array}{l}10.3 \% \\
(7.8,12.9)\end{array}$ & $\begin{array}{l}10.8 \% \\
(7.3,14.5)\end{array}$ & $\begin{array}{l}9.8 \% \\
(7.1,12.6)\end{array}$ \\
\hline & P4-P7 & $\begin{array}{l}65.3 \% \\
(61.7,68.9)\end{array}$ & $\begin{array}{l}56.9 \% \\
(54.3 \\
59.5)\end{array}$ & $\begin{array}{l}60.0 \% \\
(56.4,63.6)\end{array}$ & $\begin{array}{l}57.9 \% \\
(55.2,60.7)\end{array}$ \\
\hline & $\begin{array}{l}\text { Any } \\
\text { secondary }\end{array}$ & $\begin{array}{l}15.9 \% \\
(12.3,19.5)\end{array}$ & $\begin{array}{l}20.4 \% \\
(17.8 \\
23.0)\end{array}$ & $\begin{array}{l}18.0 \% \\
(14.4,21.6)\end{array}$ & $\begin{array}{l}19.1 \% \\
(16.5,22)\end{array}$ \\
\hline & $\begin{array}{l}\text { Any } \\
\text { postsecondary }\end{array}$ & $\begin{array}{l}1.1 \% \\
(0,4.7)\end{array}$ & $\begin{array}{l}1.6 \% \\
(0,4.2)\end{array}$ & $\begin{array}{l}3.0 \% \\
(0,6.6)\end{array}$ & $\begin{array}{l}3.3 \% \\
(0.6,6.1)\end{array}$ \\
\hline & $\begin{array}{l}\text { Attended } \\
\text { school; level } \\
\text { not specified }\end{array}$ & $\begin{array}{l}1.1 \% \\
(0,4.7)\end{array}$ & $\begin{array}{l}0.6 \% \\
(0,3.2)\end{array}$ & $\begin{array}{l}0.1 \% \\
(0,3.8)\end{array}$ & $\begin{array}{l}0.7 \% \\
(0,3.5)\end{array}$ \\
\hline
\end{tabular}

${ }^{1}$ Confidence intervals shown in parentheses

Table 3. Household survey results: proportion of 'appropriate'** treatment for recent illness in children under five years old by maternal report.

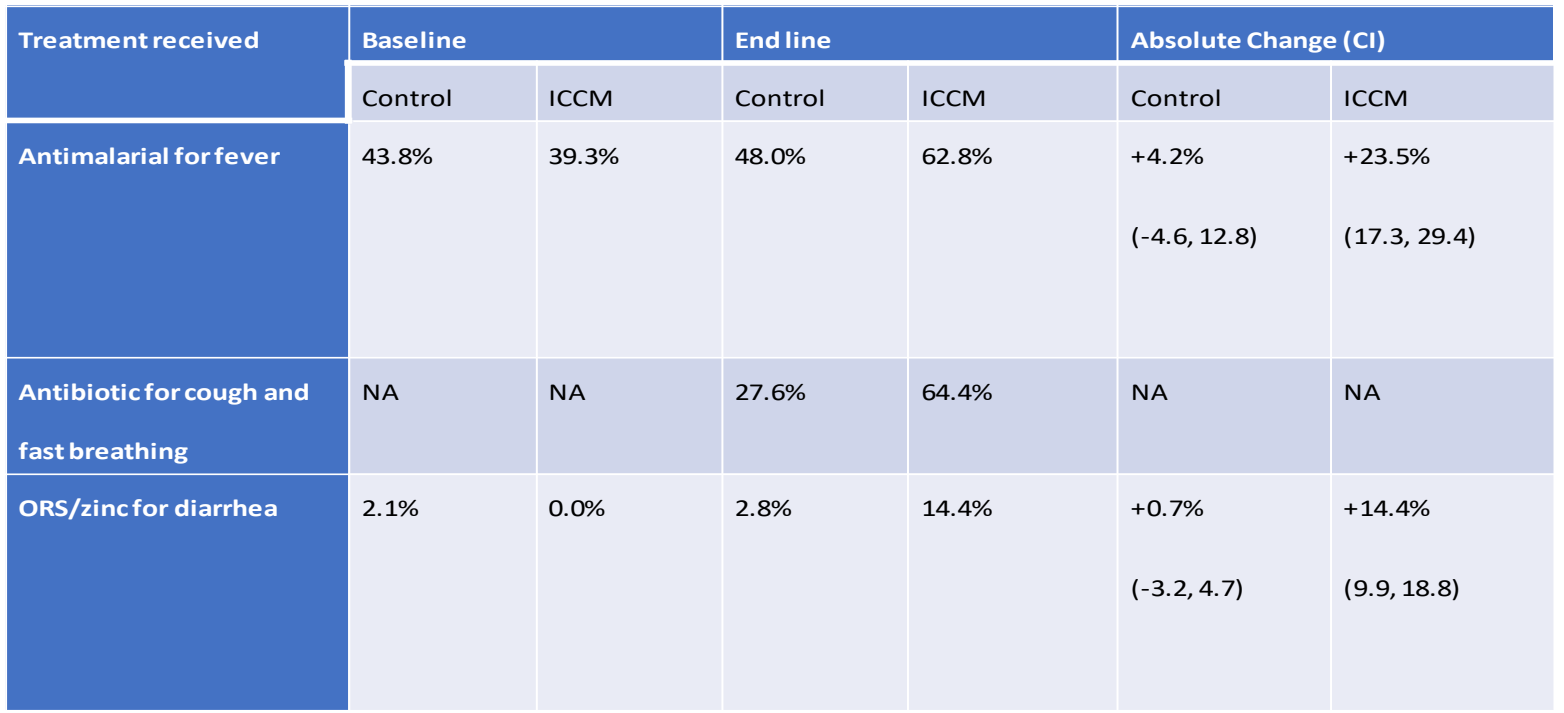

Note: Questions were asked to mothers about recent (past two week) illness in the two youngest children under five years old living in the household at time of survey. All cases of illness were analyzed separately; a single child may have been reported to have more than one category of illness.

***Treatment was determined to be 'appropriate' if the mother reported the child received an antimalarial for fever (chloroquine or sulfadoxine/pyrimethamine alone were not considered acceptable), an antibiotic for cough and fast breathing, ORS and zinc for diarrhoea. Dosing, duration and age range were not considered.

\section{Discussion}

During a short implementation period in our rural Ugandan setting, volunteer CHWs trained over short duration and supervised by local government health workers were willing and able to provide iCCM. In the communities they served, a significantly higher proportion of mothers of sick children reported their child received treatment for fever and diarrhoea; a pattern not observed in a comparison non-intervention group.
Patient encounter reports by CHWs suggest that community members are willing to access care through volunteer CHWs in this setting. Improved access to treatment for young children with malaria, pneumonia and diarrhea can translate into lives saved in rural Uganda. Where falciparum malaria is endemic and a leading cause of child deaths ${ }^{34}$, iCCM uptake could potentially reduce mortality. Country-wide, pneumonia often goes undertreated and is thought to be a major contributor to child mortality ${ }^{35}$; in- 
creased antibiotic treatment through iCCM could significantly improve survival. Increased ORS/Zinc is effective especially if those treated represent the most severe cases. In our study, diarrhoea treatment rates were too low to speculate on the importance of its inclusion as part of an iCCM package. Further studies in similar communities assessing use of ORS/zinc focused on more severe cases of diarrhoea (i.e. moderate/severe dehydration, at highest risk of death) might better assess the potential impact of its inclusion.

Global iCCM interest has increased since joint WHO/ UNICEF statements emphasized the importance of community-based management for pneumonia and diarrhoea almost a decade ago. ${ }^{36,37}$ As governments in sub-Saharan Africa are now scaling up iCCM programs, ${ }^{4}$ some peer-reviewed intervention studies are beginning to show promising results. Rwanda saw a significant decline in child mortality following nationwide iCCM implementation. ${ }^{8}$ In Ghana, management of fever cases with an antimalarial and anti-biotic demonstrated decreased mortality in a cluster randomized control trial. ${ }^{21}$ A Zambian cluster-randomized trial combining pneumonia CCM with rapid diagnostic malaria testing and empiric anti-malarial treatment for fever demonstrated promising outcomes. ${ }^{20}$ In a multi-methods Ethiopian study, volunteer CHWs treating diarrhoea, pneumonia, and fever and were highly accessed by community members ${ }^{22}$. Our study is complimentary suggesting multi-illness iCCM using $\mathrm{CHWs}$ with variable formal education is feasible, which is supportive of work in Eastern Uganda ${ }^{23,27-30}$; however, caution is needed to further understand quality of work, an issue highlighted by a recent study from Central Uganda. ${ }^{38}$

A strength of this implementation study was existing government health structure integration, which demonstrated real-life iCCM-programming feasibility and outcomes. Decision-makers were key in planning, procuring drug supplies, and district and national health professionals provided training and $\mathrm{CHW}$ support. Challenges experienced by our team including drug procurement, access to training materials, and approval for use of new algorithms represent real-life hurdles in translating policy to action. Although initial selection, training, supervision and equipping of iCCM CHWs is feasible with good planning and support, our experiences should also serve as caution for planning larger and longer implementation. The real-life challenges we faced are also well described by others ${ }^{9,38}$ and can be partly mitigated by careful plan- ning, support, and adequate long-term funding at the national level prior to broader scale-up.

This study's ultimately very short intervention period creates a major limitation in interpreting results. A longer study period could better assess seasonal activity variation, CHW retention, competency, maintenance, supply chain management and medium-term outcomes. The small and localized population covered by our intervention may have resulted in experiences not generalizable for national iCCM scale-up. In our setting, a small population with strong pre-existing community and health leader support in a single geographical area was carefully monitored and supervised. Less engaged communities, different cultural groups, families living closer to health facilities, and urban/semi-urban populations may prefer alternate care providers.

CHW-reported illness treatment was used as a proxy to approximate service usage and illness distribution. Self-reporting is subject to error including assessment and purposeful/accidental documentation especially where training on reporting and formal education levels are relatively minimal. Thus, this data should only be interpreted as an overall reflection of uptake of use by community members and distribution of caseload by classified illness. Although we did not have the capacity to do so in this study, objective CHW observations and timely and regular audits of encounters would help better understand CHW capacities and limitations for care and reporting in future evaluations.

Regrettably, during most of the intervention period, our control group had no trained CHWs due to failure of planned district programming in collaboration with development partners. Therefore, we could not differentiate the CHW 'treatment' role from the basic health promotion and referral-only role. Further quantitative and qualitative research to assess the longer-term health outcomes from curative versus promotion-only roles would be important.

While we report initial iCCM success in improving care access, further objective iCCM study in a larger, controlled trial including a health economic assessment is imperative. How do short and longer-term iCCM costs/benefits compare to health facility-based strengthening, which may also impact health access? Should iCCM be equally rolled out nationally or prioritized to remote communi- 
ties? Will iCCM encourage drug 'overtreatment', resulting in drug resistance? Addition of rapid diagnostic testing to iCCM (proven feasible elsewhere in Uganda and sub-Saharan Africa) $)^{20,29,39}$ should be seriously considered.

\section{Conclusion}

The critical health worker shortage in sub-Saharan Africa will continue for the foreseeable future. For many children, access to simple life-saving treatments for malaria, pneumonia, and diarrhoea will remain difficult. Our implementation study adds to growing iCCM literature showing feasibility for illness management by volunteer CHWs equipped with basic drugs and minimal training. In Africa, iCCM is a new and ambitious strategy. Phased introduction, careful attention to operational details, and implementation research ${ }^{37}$ will improve scale-up success. Plans for ongoing supervision, financial and non-financial incentives, and drug supply should be carefully addressed before large-scale implementation. Future iCCM programs will require significant inputs including resources, stakeholder commitment, and well-designed plans, and should not be a low-cost replacement for increasing skilled health workers in underserved areas, but rather complementary during transition to a larger skilled workforce. How CHWs can maintain their key health promotion role is critical. To maximize child survival, iCCM, which can provide vulnerable children with simple and inexpensive treatments to save their lives, must be complemented by strong community-based health promotion to prevent illnesses from even occurring.

\section{Conflict of interest}

The authors declare the following: JLB, CB, SM, JK, ANA, DB, TK, CP, KW and NS received reimbursement for travel costs associated with this study from the Global Health Research Initiative (GHRI)(Grant 105957-001) administered through the International Development Research Centre and funded with support from the Government of Canada through the Department of Foreign Affairs, Trade and Development. TK served as project manager for Healthy Child Uganda during the study(salary support through Healthy Generations Foundation); NM was employed through GHRI grant funding as project coordinator; and DB receive post-doctoral funding support through the GHRI grant.

\section{Acknowledgements}

This study was through the Global Health Research Ini- tiative (Grant 105957-001) with generous support from the International Development Research Centre and Canadian Institutes of Health Research with funding from the Government of Canada through the Department of Foreign Affairs, Trade and Development.

We thank CHWs, health workers from Bushenyi District Health Services (Dr. Edward Mwesigye, Anthony Nimukama), community members, partners (UNICEF), and Mbarara University of Science and Technology faculty/ staff (Moses Ntaro), and Ugandan Ministry of Health leaders (Dr. JescaNsungwaSabiiti). Also thanks to the many research assistants (Gad Agaba, TonnyEngwau, David Tumusiime, Polar Rwandekeye, Bob Rwabijaju, Kevin Areeba, Edmund Akathuhamya, John Baptist Lwangaand IngunnBenediktsson). Special thanks to Hannah Faye Mercader for data management support and technical manuscript assistance.

\section{References}

1. Bhutta ZA, Black RE. Global maternal, newborn, and child health--so near and yet so far. N EnglJ Med. 2013 Dec 5;369(23):2226-2235. doi: 10.1056/NEJMra1111853.

2. Global Health Workforce Alliance, World Health Organization. Global experience of community health workers for delivery of health related Millennium Development Goals: a systematic review, country case studies, and recommendations for integration into national health systems. Geneva: World Health Organization; 2010.

3. Young M, Wolfheim C, Marsh DR, Hammamy D. World Health Organization/United Nations Children's Fund joint statement on integrated community case management: an equity-focused strategy to improve access to essential treatment services for children. Am J Trop Med Hyg. 2012 Nov;87(5 Suppl):6-10. doi: 10.4269/ajtmh.2012.12-0221.

4. George A, Young M, Nefdt R, Basu R, Sylla M, Clarysse $\mathrm{G}$, et al. Community health workers providing government community case management for child survival in sub-Saharan Africa: who are they and what are they expected to do? Am J Trop Med Hyg. 2012 Nov;87(5 Suppl):85-91. doi: 10.4269/ajtmh.2012.11-0757.

5. Nsona H, Mtimuni A, Daelmans B, Callaghan-Koru JA, Gilroy K, Mgalula L, et al. Scaling up integrated community case management of childhood illness: update from Malawi. Am J Trop Med Hyg. 2012 Nov;87(5 Suppl):54-60. doi: 10.4269/ajtmh.2012.11-0759.

6. Government of Uganda Ministry of Health. Integrat- 
ed community case management of childhood malaria, pneumonia and diarrhoea: implementation guidelines. Kampala: Government of Uganda; 2010 May.

7. Marsh DR, Nefdt R, Hazel E. Introduction to a special supplement: delivering integrated community case management to treat childhood illness at scale in Ethiopia. Ethiop Med J. 2014 Oct;52 Suppl 3:1-6.

8. Mugeni C, Levine AC, Munyaneza RM, Mulindahabi E, Cockrell HC, Glavis-Bloom J, et al. Nationwide implementation of integrated community case management of childhood illness in Rwanda. Glob Health Sci Pract. 2014 Aug 5;2(3):328-341. doi: 10.9745/GHSP-D-14-00080 [doi].

9. Daniels K, Sanders D, Daviaud E, Doherty T. Valuing and Sustaining (or Not) the Ability of Volunteer Community Health Workers to Deliver Integrated Community Case Management in Northern Ghana: A Qualitative Study. PLoS One. 2015 Jun 16;10(6):e0126322. doi: 10.1371/journal.pone.0126322 [doi].

10. CORE Group, Save the Children, BASICS, MCHIP. Community case management essentials: treating common childhood illnesses in the community, a guide for program managers. Washington, D.C.: U.S. Agency for International Development; 2010.

11. Perry H, Freeman P, Gupta S, Rassekh BM. How effective is community-based primary health care in improving the health of children? Summary findings: report to the expert review panel. Washington, D.C.: American Public Health Association; 2009.

12. Haines A, Sanders D, Lehmann U, Rowe AK, Lawn JE, Jan S, et al. Achieving child survival goals: potential contribution of community health workers. Lancet. 2007 Jun 23;369(9579):2121-2131. doi: 10.1016/S01406736(07)60325-0.

13. United Nations Children's Fund, World Health Organization. Management of sick children by community health workers: intervention models and programme examples. New York: United Nations Children's Fund; 2006.

14. Laínez YB, Wittcoff A, Mohamud AI, Amendola P, Perry HB, D'Harcourt E. Insights from community case management data in six sub-Saharan African countries. Am J Trop Med Hyg. 2012 Nov;87(5 Suppl):144-150. doi: 10.4269/ajtmh.2012.12-0106.

15. Philips M, Zachariah R, Venis S. Task shifting for antiretroviral treatment delivery in sub-Saharan Africa: not a panacea. Lancet. 2008 Feb 23;371(9613):682-684. doi: 10.1016/S0140-6736(08)60307-4.
16. Bari A, Sadruddin S, Khan A, Khan I, Khan A, Lehri IA, et al. Community case management of severe pneumonia with oral amoxicillin in children aged 2-59 months in Haripur district, Pakistan: a cluster randomised trial. Lancet. 2011 Nov 19;378(9805):1796-1803. doi: 10.1016/ S0140-6736(11)61140-9.

17. Ghimire M, Pradhan YV, Maskey MK. Community-based interventions for diarrhoeal diseases and acute respiratory infections in Nepal. Bull World Health Organ. 2010 Mar;88(3):216-221. doi: 10.2471/BLT.09.065649.

18. Druetz T, Siekmans K, Goossens S, Ridde V, Haddad $\mathrm{S}$. The community case management of pneumonia in Africa: a review of the evidence. Health Policy Plan. 2015 Mar;30(2):253-266. doi: 10.1093/heapol/czt104.

19. Kelly JM, Osamba B, Garg RM, Hamel MJ, Lewis JJ, Rowe SY, et al. Community health workerperformance in the management of multiple childhood illnesses: Siaya District, Kenya, 1997-2001. Am J Public Health. 2001 Oct;91(10):1617-1624.

20. Yeboah-Antwi K, Pilingana P, Macleod WB, Semrau $\mathrm{K}$, Siazeele K, Kalesha P, et al. Community case management of fever due to malaria and pneumonia in children under five in Zambia: a cluster randomized controlled trial. PLoS Med. 2010 Sep 21;7(9):e1000340. doi: 10.1371/ journal.pmed.1000340.

21. Chinbuah MA, Kager PA, Abbey M, Gyapong M, Awini E, Nonvignon J, et al. Impact of community management of fever (using antimalarials with or without antibiotics) on childhood mortality: a cluster-randomized controlled trial in Ghana. Am J Trop Med Hyg. 2012 Nov;87(5 Suppl):11-20. doi: 10.4269/ajtmh.2012.12-0078.

22. Degefie T, Marsh D, Gebremariam A, Tefera W, Osborn $\mathrm{G}$, Waltensperger K. Community case management improves use of treatment for childhood diarrhea, malaria and pneumonia in a remote district of Ethiopia. Ethiop J Health Dev. 2009;23(2):120-126.

23. Kalyango JN, Alfven T, Peterson S, Mugenyi K, Karamagi C, Rutebemberwa E. Integrated community case management of malaria and pneumonia increases prompt and appropriate treatment for pneumonia symptoms in children under five years in Eastern Uganda. Malar J. 2013 Sep 22;12:340. doi: 10.1186/1475-2875-12-340.

24. Nsungwa-Sabiiti J, Peterson S, Pariyo G, Ogwal-Okeng J, Petzold MG, Tomson G. Home-based management of fever and malaria treatment practices in Uganda. Trans R Soc Trop Med Hyg. 2007 Dec;101(12):1199-1207. doi: 10.1016/j.trstmh.2007.08.005.

25. Government of Uganda Ministry of Health. Health 
sector strategic plan III: 2010/11-2014/15. Kampala: Government of Uganda; 2010.

26. Government of Uganda Ministry of Health. Village Health Team strategy and operational guidelines. Kampala: Government of Uganda; 2010 March.

27. Kalyango JN, Lindstrand A, Rutebemberwa E, Ssali S, Kadobera D, Karamagi C, et al. Increased use of community medicine distributors and rational use of drugs in children less than five years of age in Uganda caused by integrated community case management of fever. $A m \mathrm{~J}$ Trop Med Hyg. 2012 Nov;87(5 Suppl):36-45. doi: 10.4269/ ajtmh.2012.11-0733.

28. Rutebemberwa E, Kadobera D, Katureebe S, Kalyango JN, Mworozi E, Pariyo G. Use of community health workers for management of malaria and pneumonia in urban and rural areas in eastern Uganda. Am J Trop Med Hyg. 2012 Nov;87(5 Suppl):30-35. doi: 10.4269/ajtmh.2012.11-0732.

29. Kalyango JN, Rutebemberwa E, Alfven T, Ssali S, Peterson S, Karamagi C. Performance of community health workers under integrated community case management of childhood illnesses in eastern Uganda. Malar J. 2012 Aug 20;11:282. doi: 10.1186/1475-2875-11-282.

30. Kalyango JN, Rutebemberwa E, Karamagi C, Mworozi E, Ssali S, Alfven T, et al. High adherence to antimalarials and antibiotics under integrated community case management of illness in children less than five years in eastern Uganda. PLoS One. 2013;8(3): PubMed e60481. doi: $10.1371 /$ journal.pone.0060481.

31. Government of Uganda Bushenyi District Health Services. Bushenyi District Health Records; 2010 (Unpublished).

32. Tumusiime D, Agaba G, Kyomuhangi T, Finch J, Kabakyenga J, MacLeod S. Introduction of mobile phones for use by volunteer community health workers in support of integrated community case management in Bushenyi District, Uganda: development and implementation process. BMC Health Serv Res. 2014;14(Suppl 1):S2. doi: 10.1186/1472-6963-14-S1-S2.

33. Kabakyenga J, Barigye C, Brenner J, Maling S, Buchner D, Nettel-Aguirre A, et al. A demonstration of mobile phone deployment to support the treatment of acutely ill children under five in Bushenyi District, Uganda.

Afr Health Sci. Accepted.

34. Brenner JL, McElroy T, Kayizzi J, O’Connell C, Joseph KS, Smart K, et al. Mothers report high child mortality and large burden of illness in rural Uganda. Paediatr Child Health 2007 May/June;12(A Suppl).

35. Government of Uganda Ministry of Health. Child survival strategy for Uganda 2008-2015. Kampala: Government of Uganda; 2007.

36. World Health Organization, United Nations Children's Fund. Management of pneumonia in community settings: WHO/UNICEF joint statement. Geneva: World Health Organization; 2004.

37. World Health Organization, United Nations Children's Fund. Clinical management of acute diarrhoea: WHO/UNICEF joint statement. Geneva: World Health Organization; 2004.

38. Bagonza J, Kibira SP, Rutebemberwa E. Performance of community health workers managing malaria, pneumonia and diarrhoea under the community case management programme in central Uganda: a cross sectional study. Malar J. 2014 Sep 18;13:367-2875-13-367. doi: 10.1186/1475-2875-13-367 [doi].

39. Mukanga D, Tibenderana JK, Peterson S, Pariyo GW, Kiguli J, Waiswa P, et al. Access, acceptability and utilization of community health workers using diagnostics for case management of fever in Ugandan children: a cross-sectional study. Malar J. 2012 May 24;11:121. doi: 10.1186/1475-2875-11-121. 